\title{
Medición de usabilidad y portabilidad de una Aplicación Web desarrollada con tecnología PWA
}

\section{Measurement of usability and portability of a web application developed with PWA technology}

Yessenia Karolina Macías Guamangate. ${ }^{1}$, José Leonardo Miranda Caisaluisa. ${ }^{2}$ \& Verónica del Consuelo Tapia Cerda. ${ }^{3}$

\begin{abstract}
. DOI: https://doi.org/10.33262/concienciadigital.v4i4.1882

Introduction: PWAs are web applications considered as a natural evolution of traditional web applications, capable of integrating into mobile devices and performing tasks that generally only native applications could perform. Usability and portability correspond to two parameters of the quality model, usability refers to the ease with which people can use a computer tool. It is based on three parameters: efficiency, effectiveness and satisfaction. On the other hand, portability is defined as the effort to transfer the application to another operating system, device and platform. Objective. To measure the usability and portability parameters in the Traditional Web Application and the Progressive Web Application (PWA), created for a home delivery service, through the use of the questionnaire called System Usability Scale (SUS) and the use of general formulas in order to obtain information and perform a comparative analysis of the results. Methodology. The present research is defined as comparative descriptive, it is part of the

\footnotetext{
${ }^{1}$ Universidad Técnica de Cotopaxi, Facultad de Ciencias de la Ingeniería y Aplicadas. Latacunga, Ecuador. yessenia.macias6708@utc.edu.ec. ORCID https://orcid.org/0000-0002-0422-6731

${ }^{2}$ Universidad Técnica de Cotopaxi, Facultad de Ciencias de la Ingeniería y Aplicadas. Latacunga, Ecuador. jose.miranda7348@utc.edu.ec. ORCID https://orcid.org/0000-0001-9799-5283

${ }^{3}$ Universidad Técnica de Cotopaxi, Facultad de Ciencias de la Ingeniería y Aplicadas. Latacunga, Ecuador. veronica.tapia@utc.edu.ec ORCID https://orcid.org/0000-0002-5338-7814
} 
quantitative paradigm; it uses the survey and formulas in the measurement process, as well as the Iterative - Incremental model for the development of web applications. The population consisted of 25 users to whom the SUS usability questionnaire validated by the scientific community that studies usability issues was applied. Results. The results reveal that the Usability in the traditional web application is 80.3 , while the PWA presents an overall average result of 84.02 ; in portability the values are between $75 \%$ and $85.35 \%$ respectively. Conclusions. It is concluded that in terms of usability and portability the values favor the PWA, a result that is mainly attributed to the improvement characteristics that this technology presents in the development of web applications.

Keywords: usability, portability, software development, progressive web applications.

\section{Resumen.}

Introducción: Las PWA son aplicaciones web consideradas como una evolución natural de las aplicaciones web tradicionales, capaces de integrarse en dispositivos móviles y realizar tareas que generalmente solo las aplicaciones nativas podían llevar a cabo. La usabilidad y portabilidad corresponden a dos parámetros del modelo de calidad, la usabilidad hace referencia a la facilidad con que las personas pueden utilizar una herramienta informática. Se basa en tres parámetros: eficiencia, eficacia y satisfacción. Por otro lado, la portabilidad se define como el esfuerzo para transferir la aplicación a otro sistema operativo, dispositivo o plataforma. Objetivo. Medir los parámetros de usabilidad y portabilidad en la Aplicación Web Tradicional y la Aplicación Web Progresiva (PWA), creadas para un servicio de entrega a domicilio, mediante el uso del cuestionario denominado System Usability Scale (SUS) y la utilización de fórmulas generales con la finalidad de obtener información y realizar el análisis comparativo de los resultados. Metodología. La presente investigación se define como descriptiva comparativa, se inscribe en el paradigma cuantitativo; emplea la encuesta y fórmulas en el proceso de medición, así como el modelo Iterativo - Incremental para el desarrollo de las aplicaciones web. La población fue constituida por 25 usuarios a quienes se aplicó el cuestionario de usabilidad SUS validado por la comunidad científica que estudia temas de usabilidad. Resultados. Los resultados revelan que la Usabilidad en la aplicación web tradicional es de 80.3 , mientras que la PWA presenta un resultado promedio global de 84.02; en portabilidad los valores se encuentran entre un $75 \%$ y un $85.35 \%$ respectivamente. Conclusiones. Se concluye que en cuanto a usabilidad y portabilidad los valores favorecen a la PWA, resultado que se atribuye principalmente a las características de mejora que presenta esta tecnología en el desarrollo de aplicaciones web.

Palabras claves: usabilidad, portabilidad, desarrollo de software, aplicaciones web progresivas. 


\section{Introducción.}

En la actualidad el uso de las TICs se ha vuelto un aliado imprescindible para las personas por el sinnúmero de beneficios que ofrecen las aplicaciones web; entre ellos que combinadas con el internet, facilitan el acceso a la información y la realización de diversas operaciones (R. A. Rodríguez et al., 2020); sin embargo hay una brecha entre las capacidades de las aplicaciones nativas sobre las web, reducida por los estándares web de acceso al hardware (R. A. Rodríguez et al., 2019). Limitaciones que impiden una satisfacción del usuario. Basado en éste y otros enfoques aparece la tecnología Aplicaciones Web Progresivas:

"una evolución natural de las aplicaciones web que difuminan la barrera entre la web y las aplicaciones, capaces de integrarse en los dispositivos móviles pudiendo realizar tareas que generalmente solo las aplicaciones nativas podían llevar a cabo” (P. Rodríguez, 2018),

En la definición de las PWA se involucran un conjunto de conceptos y palabras clave que incluyen progresivo, responsivo, conectividad, independiente, segura, descubrible, instalable y enlazable(Biørn-hansen et al., 2017).

Integraciones donde se accede a tecnologías de bajo nivel, al respecto Rodríguez (2018) afirma que la web debería ser nuestro medio principal de consumo de aplicaciones en estos entornos (p. 1), pero no es así porque para los usuarios desinformados hay desventajas, a pesar de recibir notificaciones push; agregarse a la pantalla de inicio y al buscador de aplicaciones (p. 1), entre otras. La problemática entre aplicaciones web y nativas que Rodríguez aprecia determinadas por el uso frente a la capacidad de descubrimiento; usuarios activos, frente a alcance de nuevo público (p.1) que provocan sea difícil distinguir la aplicación que utiliza y la posibilidad de selección.

En el contexto anterior, este trabajo se propone evaluar la usabilidad y portabilidad de la Aplicación Web de servicios de entrega a domicilio que se desarrolla por la entidad VeciEntrega en el cantón Latacunga - Ecuador para el perfeccionamiento y agilidad de las actividades demandadas por el servicio.

En 2015 el ingeniero de Google Chrome Alex Russell y la diseñadora Frances Berriman introdujeron el concepto de Progressive Web App(PWA) significó la instalación en una plataforma muy empleada, aunque otras la integren: IOS, Android, Windows, macOS y Linux. El concepto de PWA se acuño "para describir las aplicaciones que aprovechan las nuevas características admitidas por los navegadores modernos, como los services workers y los manifiestos de app manifests" (Tandel, 2018), archivos que permiten convertir las apps web en aplicaciones web progresivas en su sistema operativo (OS) nativo.(Tandel, 2018). A su vez, significó una solución renovadora en tecnología; tanto es así que establece algunos requisitos para que una Aplicación Web sea considerada una PWA:

a) funcionamiento offline (trabajar sin necesidad de conexión a internet), 
b) brindar experiencia de uso similar a una aplicación nativa,

c) utilizar el protocolo HTTPS (R. A. Rodríguez et al., 2019).

El último requisito se establece como una condición obligatoria de las PWA, su conexión con el servidor debe producirse mediante este protocolo y que toda petición que intente realizarse mediante HTTP se redirija a su equivalente seguro (P. Rodríguez, 2018).

Algunas tecnologías y conceptos que aplica las PWA son:

$>$ Services Workers o SW, es un archivo JavaScript que incorpora ganchos de ciclo de vida para la lógica de negocio y control de caché, se utiliza para gestionar tareas como la sincronización en segundo plano, mecanismos de caché para datos y Shell de aplicaciones, así como la interceptación de solicitudes de red(Biørn-hansen et al., 2017). El tiempo de vida de los SW se mantienen generalmente por un corto tiempo. Se despierta cuando recibe un evento y se ejecuta sólo el tiempo que necesita para procesarlo(Mhaske et al., 2018).

$>$ Application Shell, según Osmani y Gaunt (2017) "es el mínimo de HTML, CSS y JavaScript que alimentan una interfaz de usuario". Enumeran tres criterios para la shell: tiempo de carga rápido, almacenamiento en caché y visualización de contenido dinámico(Sharma et al., 2019).

$>$ Web App Manifest, es un lugar para almacenar los metadatos de la App(Karim, 2016), su propósito es exponer ciertos configuraciones modificables a los desarrolladores(Sharma et al., 2019) como la ruta del logotipo, el nombre de la aplicación, etc.

$>$ El SW es la columna vertebral de la PWA. Almacena la memoria y ayuda a añadir accesibilidad y funcionalidad sin conexión(Khan et al., 2019), por tanto que junto con el App Manifest se consideran dos componentes principales en una PWA.

Algunas características definidas para las PWA en (Steiner, 2018) son:

Capacidad en línea, seguir trabajando con ciertas funcionalidades incluso cuando el dispositivo esta fuera de línea; capacidad de mostrar notificaciones push; capacidad de instalarse a la pantalla de inicio de un dispositivo; capacidad de sincronizar datos en segundo plano; capacidad de almacenar datos de forma persistente; capacidad de mostrar metadatos multimedia personalizados en la interfaz de usuario de la plataforma; capacidad de tomar una decisión óptima a la hora de elegir el contenido multimedia para el usuario, entre otras.

Las tecnologías PWA facilitan el empleo de mecanismos que superan limitaciones correspondientes al enfoque web móvil y con esto mayor calidad; la cual se define como grado de satisfacción de las necesidades implícitas y explícitas cuando esta es utilizada bajo ciertas condiciones (Reyes et al., 2016).

El modelo de calidad del software se encuentra establecido por los estándares ISO/IEC 9126-1(Mittal \& Kumar Bhatia, 2013) y su actualización en la ISO/IEC 25010 (ISO, 
2019), actualmente está definido por ocho características donde la usabilidad y la portabilidad son parte de ellas.

La portabilidad en tanto es una condición de las tecnologías Web, la ISO/IEC 25010 la define como:

"Capacidad del producto o componente de ser transferido de forma efectiva y eficiente de un entorno hardware, software, operacional o de utilización a otro.”(ISO, 2019)

La esencia que la configura es la facilidad de adaptación del producto a otras plataformas y dispositivos (Barbosa et al., 2020). A su vez subdivide esta característica en otras tres subcaracterísticas que incluyen; adaptabilidad, capacidad de ser instalado y la capacidad para ser reemplazado.

Se entiende que el proceso de portabilidad de un software se basa en dos componentes principales: la transportación y la adaptación; la transportación se refiere al movimiento físico y; la adaptación es cualquier modificación que deba realizarse en la versión original entendiéndose a la traducción mecánica como la realizada por procesadores lingüísticos, y la modificación manual por parte de los humanos.(Mooney, 1997)

El estándar ISO9241-11 (1998) define la usabilidad como "la medida en la que un producto se puede usar por determinados usuarios para conseguir objetivos específicos con efectividad, eficiencia y satisfacción en un contexto de uso especificado". La referencia a las cualidades a lograr fundamenta la primera como la exactitud con la cual los usuarios alcanzan sus metas; mientras que la segunda alude a recursos gastados con relación a la certeza con la cual los usuarios se sienten haber alcanzado sus propósitos; a su vez la satisfacción comprende la comodidad y aceptabilidad del uso (Perurena \& Moráguez Bergues, 2013). Tales cualidades dependen de la estructura tecnológica y el diseño elementos que serán abordados como desarrolladores que resuelvan mayores niveles de usabilidad y satisfacción del usuario.

En la usabilidad web subyacen manifestaciones de incoherencias; puede ser porque los usuarios dominan escasamente metodologías subjetivas o variables básicas, o son personas mayores o personas con discapacidad (Laparra Hernández, 2015). Causas determinantes de exclusión en la Sociedad de la Información. Por ello se ha indagado la respuesta fisiológica y la mirada para la selección de la información en la interacción con la web. Los resultados explican que el modelo de valoración de usabilidad varía en función del perfil de usuario (Laparra Hernández, 2015).

La calidad de un producto software puede ser medible y variable, de aquí la existencia de un sinnúmero de métodos y herramientas según el contexto de la aplicación. Los cuestionarios, son formas exitosas para realizar este trabajo debido a la sencillez y facilidad. A pesar de que éstos se utilizan para evaluar tipos específicos de interfaces (Geraldes et al., 2019) existen otros que pueden ser aplicados. Es el caso del cuestionario SUS (System Usability Scale) creado por John Brooke en 1986 (Klug, 2017) mientras 
formaba parte de Digital Equipment Corporation (Borsci et al., 2009); la versión estándar del Sistema de Escala de Usabilidad consta de 10 ítems, cada uno con cinco pasos anclados con "Totalmente en desacuerdo" y "Totalmente de acuerdo". Se trata de un cuestionario de tono mixto(Lewis Senior HF Engineer \& Sauro, 2017) en el que los ítems impares tienen un tono positivo y los pares un tono negativo.

Sugiere ser rápido y fácil para los participantes del estudio, rentable de usar y puntuar; en cuanto tecnología independiente puede ser utilizada por un amplio grupo de profesionales en la evaluación de interfaz de usuario; con su puntaje único varía de 0 a 100, fácil de entender por una amplia gama de personas (Geraldes et al., 2019).

En el presente artículo se realizará la medición de la usabilidad y portabilidad utilizando el cuestionario SUS y las fórmulas de las métricas externas de portabilidad añadidas en la ISO/IEC 9126.

El desarrollo de las aplicaciones progresivas ha sido factible por avances tecnológicos que ilustra Pablo Rodríguez (2018) en el fragmento siguiente:

Es el estándar que actualmente domina la web y que supone la base sobre la que hoy se asientan las PWAs, ya que ofrece APIs tan variadas y necesarias como: almacenamiento local, acceso a tecnologías de bajo nivel del dispositivo, web sockets, web workers, canvas, drag and drop, svg, entre otras muchas (p. 13).

La diversidad de alcances contenida en dicha tecnología se aprecia como elemento fundamental del desarrollo de las aplicaciones PWA (Progressive Web Application), que se están erigiendo como la tendencia en la forma de acceder y servir contenido (Lanza, 2019). Ese servicio se ancla en la concepción aplicación nativa debido a que el diseño de su arquitectura funcional implementa servicios integradores de un proceso que abarca emulación de sensor, base de datos, hasta las interfaces de visualización e interacción (Lanza, 2019); lo cual constituye una respuesta funcional y de eficacia del dispositivo que se emplee. Para la portabilidad es imprescindible evaluar que la lectura se realice al ciclo de vida de una arquitectura; comprendido por los requisitos, diseño, documentación, evaluación e implementación (Bautista Peñaquishpe, 2019), en ellos corresponde determinar la funcionalidad, adaptabilidad y rendimiento; esas cualidades posee la "App Shell" como arquitectura ideal que hace factible la Web Progresiva de tipo informativo, un entorno anclado entre las aplicaciones web nativas e híbridas (p. 78). Sus características denotan el valor de cualidades centradas en adaptación, trabajar sin conexión que las visiona como la tecnología de futuro.

\section{Metodología.}

La presente investigación se define como descriptiva comparativa, se inscribe en el paradigma cuantitativo y se orienta a describir el nivel de satisfacción de los usuarios y a calcular el porcentaje de portabilidad de la Aplicación Web en sus versiones tradicional y PWA. El software fue desarrollado para prestar el servicio de entrega a domicilio en la 
provincia de Cotopaxi - Ecuador, e instalado en locales comerciales por la entidad VeciEntrega.

Para el estudio de la usabilidad la población fue conformada por personas mayores de 18 años de la ciudad de Latacunga y sus alrededores, el cálculo se realizó a través de la fórmula para población finita dando como resultado 25 usuarios, seleccionados bajo el concepto del muestreo no probabilístico intencional (Otzen \& Manterola, 2017), para lo cual se consideró los siguientes criterios de inclusión; propietarios o dueños de comercios, motorizados o personas que hayan trabajado en el servicio de reparto y personas naturales como clientes de los servicios. La selección estuvo sustentada en ser los actores donde concluye el ciclo del servicio: demandas, servicios y satisfacción.

En los procesos de medición se emplea el método de evaluación por indagación con el cuestionario como técnica y fórmulas generales definidas en la ISO/IEC 9126-2 que permiten determinan el valor de las subcaracterísticas de la portabilidad. Se seleccionó el modelo de desarrollo Iterativo - Incremental propuesto por Harlan Mills en el año 1980 (Zumba \& León, 2018) para la construcción del software; el modelo es evolutivo y se acopla a la naturaleza iterativa de hacer prototipos con los aspectos controlados y sistemáticos del modelo de cascada, se producen con mucha rapidez versiones funcionales del software (Pressman, 2010); las etapas metodológicas son: análisis, diseño, codificación y pruebas; repetidas en los ciclos definidos para el desarrollo de cada uno. El software se desarrolla bajo una serie de versiones cada vez más completas de la aplicación.

\section{Primera etapa - elaboración del software}

El software fue desarrollado en cinco ciclos que oscilan en intervalos de tiempo de 3 a 4 semanas; la lista de requisitos fue priorizada mediante el análisis Moscow (Simões \& Vázquez, 2018) y asignados a cada iteración respectivamente. Fue empleada la técnica de Casos de Uso para ilustrar el escenario principal y alternos, funcionalidades del sistema y usuarios. Posteriormente se desarrollaron los primeros entregables del sistema: módulo para el administrador, usuarios, dueños de locales y motorizados; luego se realizó la respectiva validación y corrección. La Aplicación Web ha sido desarrollada utilizando el Framework Django con el lenguaje de programación Python; también el lenguaje de marcado HTML5 y CSS para los estilos; además del leguaje JavaScript con su librería JQuery para el manejo del DOM y peticiones AJAX.

\section{Segunda etapa - adaptación de la tecnología PWA}

Para incluir las funcionalidades que ofrece la tecnología PWA en la Aplicación Web se utilizó una librería proporcionada por el framework Django denominada Djangoprogressive-web-app en su versión 0.1.1, al ser instalada incluye un archivo manifest.json y una instancia de Service Worker.

Archivo manifest.json 
Se genera una vez se instala la librería de Django, permitiendo controlar el cómo se visualiza la aplicación en un entorno que normalmente está diseñado para Apps Nativas. Las funcionalidades que cumple el archivo en la aplicación son: especificar el nombre de la App, añadir el icono de la entidad VeciEntrega y establecer algunos colores de diseño básico.

\section{Implementación del Service Worker o SW}

El SW intercepta las peticiones que el usuario realiza desde la aplicación al servidor; la primera petición HTTPS es dirigida directamente al servidor, toma la información solicitada y regresa para ser mostrada en el navegador. Mientras en segundo plano el ciclo de vida del Services Worker empieza a ejecutarse; primero se procede con el registro mediante el método ServiceWorkerContainer.register(); segundo se inicia la etapa de instalación mediante el evento install, se obtiene el script y se descargan todos los archivos registrados, este proceso solo sucede una vez; tercero comenzará el proceso de activación y después de esta etapa el Service Worker ya tiene el control de los eventos y la extracción de los archivos cuando se emita una petición al servidor.

En caso de una actualización del SW, este se instala en un segundo plano, pero permanece en estado Worker in Waiting hasta que las páginas que aún utilizan el SW antiguo se carguen completamente, después el archivo actualizado pasa a un estado Active Worker. Todo el proceso se ejecuta en el evento denominado actívate momento ideal para limpiar cachés antiguas y añadir la nueva actualización.

El push y fetch son eventos que se disparan cada vez que el usuario realiza una petición al servidor y se suman al proceso del SW de la Aplicación Web.

El funcionamiento online sucede una vez que el SW ya se está ejecutándose en el navegador y cuando capta la solicitud del usuario buscará y regresará los archivos almacenados en el caché local aún si en el momento no puede tener contacto con el servidor.

Las notificaciones push se ejecutan en un evento llamado load en el archivo JavaScript webpush,js donde se encuentran las funciones específicas para el funcionamiento de las notificaciones en los diferentes entornos y dispositivos.

\section{Tercera etapa - medición de usabilidad y portabilidad}

\section{Métrica de usabilidad}

El test SUS se caracteriza por una alta validez psicométrica que mide la facilidad de uso percibida por el usuario en aspectos de satisfacción, eficiencia y eficacia respecto al producto que utiliza. Consta de 10 afirmaciones en tono mixto que se puntúan en la escala LIKERT (Ver Tabla. 1) para representar el nivel de acuerdo. El resultado de la medición puede oscilar entre 0 y 100 puntos; cuanto más alto sea el resultado, mejor será la usabilidad del sistema.(Sensuse et al., 2017) 
Tabla 1. Valores de la Escala Likert del cuestionario SUS.

\begin{tabular}{lllclll} 
& \multicolumn{4}{c}{ Valores de la Escala Likert } \\
En desacuerdo & $\mathbf{1}$ & $\mathbf{2}$ & $\mathbf{3}$ & $\mathbf{4}$ & $\mathbf{5}$ & Completamente de acuerdo \\
\hline
\end{tabular}

Fuente: Elaboración propia.

\section{Proceso de aplicación del test}

El procedimiento general de la aplicación del test SUS se detalla a continuación; en primer lugar, se añadió al test instrucciones breves que permitan al usuario recordar que debe seleccionar una respuesta por cada pregunta y no detenerse demasiado tiempo en alguna de ellas; en segundo lugar se permitió al usuario interactuar con la Aplicación Web, después se solicitó que realice una determinada tarea que corresponda a las funcionalidades de la App; finalmente el usuario respondió el test permitiendo recopilar el valor Likert que los participantes asignaron a las preguntas, repitiendo el proceso para todas los integrantes de la muestra seleccionada.

\section{Cálculo}

Como primer paso para obtener un puntaje SUS es determinar las contribuciones de cada ítem seleccionado sabiendo que el valor oscila entre 0 (mala experiencia) y 4 (buena experiencia). La contribución de los puntos 1,3,5,7 y 9, es decir, los números impares es la posición de la escala menos 1 . Y para los puntos 2, 4, 6, 8 y 10, o números pares, la contribución será 5 menos la posición en la escala, finalmente se suman las contribuciones de cada punto y se multiplica por 2.5 obteniéndose el valor global cuyo resultado oscila entre 0 y 100. Donde 100 resalta la perfección en la usabilidad de la aplicación, 70 representa un valor intermedio y menos de 50 expresa una ineficiencia del sistema en cuanto a usabilidad. Cabe recalcar que estos valores no son porcentajes ni son significativos por sí mismos.

\section{Interpretación de resultados}

El resultado de un cuestionario SUS puede interpretarse de diferentes modos, se utilizará como guía general la Figura 1.

Figura 1 Clasificaciones de la puntuación SUS.

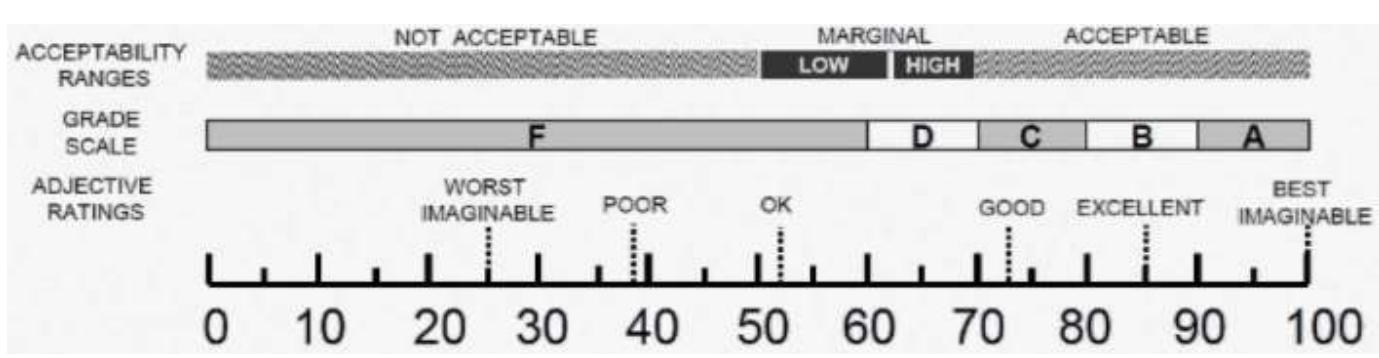

Fuente: A.Bangor, P.T Kortum, and J.T. Miller, 2009 (Bangor et al., 2009).

\section{Medición de portabilidad}

El valor estimado de portabilidad se basa en las subcaracterísticas de la portabilidad definidas en la ISO/IEC 25010:2011 (ISO, 2019) y utilizando las fórmulas de las métricas 
externas de portabilidad añadidas en la ISO/IEC 9126 que hacen referencia principalmente a la adaptabilidad e instalabilidad. Los parámetros a medir se muestran en la Tabla. 2.

Tabla 2. Características y métricas relacionadas con la portabilidad.

\section{Portabilidad}

\begin{tabular}{l|l}
\hline Subcaracterísticas de la ISO/IEC & Métricas de la ISO/IEC 9126 \\
$\mathbf{2 5 0 1 0 : 2 0 1 1}$ & \\
\hline Adaptabilidad & Adaptabilidad a distintos dispositivos \\
\hline Capacidad para ser instalado. & Facilidad de instalación \\
\cline { 2 - 2 } & Facilidad de reinstalación \\
\hline Capacidad para ser reemplazado & Uso continuado de los datos \\
\hline
\end{tabular}

Fuente: Elaboración propia.

En la Tabla. 3 se encuentran las tres subcaracterísticas de la portabilidad, las métricas relacionadas y sus respectivas fórmulas utilizadas para calcular el valor estimado de portabilidad de la Aplicaciones Web en sus dos versiones. Las métricas se encuentran en la Guía del estándar ISO 9126 acerca de la gestión de la calidad de software.

Tabla 3. Factores, métricas y fórmulas aplicadas en la medición de portabilidad,

\begin{tabular}{|c|c|c|c|}
\hline $\begin{array}{l}\text { Factor de } \\
\text { portabilidad }\end{array}$ & Métricas & Fórmula & Valor de X \\
\hline Adaptabilidad & $\begin{array}{l}\text { Adaptabilidad } \\
\text { a distintos } \\
\text { dispositivos }\end{array}$ & $\begin{array}{l}\mathrm{X}=1-\mathrm{A} / \mathrm{B} \\
\mathrm{B}=\text { número de dispositivos } \\
\text { especificados en los que el producto } \\
\text { software debe ser adaptable. } \\
\mathrm{A}=\text { número de dispositivos en los } \\
\text { que la adaptabilidad no es del todo } \\
\text { satisfactoria }\end{array}$ & $\begin{array}{l}0 \leq X \leq 1 \\
\text { Cuanto más } \\
\text { cercano a } 1 \text {, } \\
\text { mejor. }\end{array}$ \\
\hline \multirow[t]{2}{*}{$\begin{array}{l}\text { Capacidad } \\
\text { para ser } \\
\text { instalado }\end{array}$} & $\begin{array}{l}\text { Facilidad de } \\
\text { instalación }\end{array}$ & $\begin{array}{l}\mathrm{X}=\mathrm{A} / \mathrm{B} \\
\mathrm{A}=\text { Número de veces que el usuario } \\
\text { ha tenido éxito en cambiar la } \\
\text { instalación. } \\
\mathrm{B}=\text { Número de veces que el usuario } \\
\text { lo ha intentado, hayan tenido éxito o } \\
\text { no. }\end{array}$ & $\begin{array}{l}0 \leq X \leq 1 \\
\text { Cuanto más } \\
\text { cercano a } 1, \\
\text { mejor. }\end{array}$ \\
\hline & $\begin{array}{l}\text { Facilidad de } \\
\text { reinstalación }\end{array}$ & $\begin{array}{l}\mathrm{X}=1-\mathrm{A} / \mathrm{B} \\
\mathrm{A}=\text { Número de fallos del usuario al } \\
\text { intentar reinstalar el software. } \\
\mathrm{B}=\text { Número de intentos. }\end{array}$ & $\begin{array}{l}0 \leq X \leq 1 \\
\text { Cuanto más } \\
\text { cercano a } 1, \\
\text { mejor. }\end{array}$ \\
\hline $\begin{array}{l}\text { Capacidad } \\
\text { para ser } \\
\text { reemplazado }\end{array}$ & $\begin{array}{l}\text { Uso } \\
\text { continuado de } \\
\text { los datos }\end{array}$ & $\begin{array}{l}\mathrm{X}=\mathrm{A} / \mathrm{B} \\
\mathrm{A}=\text { Número de datos que se usaban } \\
\text { en el software anterior y que se }\end{array}$ & $0 \leq X \leq 1$ \\
\hline
\end{tabular}


Tabla 3. Factores, métricas y fórmulas aplicadas en la medición de portabilidad, (continuación)

\begin{tabular}{|c|c|c|c|}
\hline $\begin{array}{l}\text { Factor de } \\
\text { portabilidad }\end{array}$ & Métricas & Fórmula & Valor de X \\
\hline $\begin{array}{l}\text { Capacidad } \\
\text { para ser } \\
\text { reemplazado }\end{array}$ & $\begin{array}{l}\text { Uso } \\
\text { continuado } \\
\text { de los datos }\end{array}$ & $\begin{array}{l}\text { pueden seguir utilizando en el nuevo } \\
\text { software. } \\
\text { B = Número de datos que se usaban } \\
\text { en el software anterior y de los } \\
\text { cuales se planea su reutilización en } \\
\text { el nuevo software. }\end{array}$ & $\begin{array}{l}\text { Cuanto más } \\
\text { cercano a } 1 \text {, } \\
\text { mejor. }\end{array}$ \\
\hline
\end{tabular}

Fuente: Elaboración propia.

La Tabla. 4 muestra los subcriterios de la Adaptabilidad a ser considerados en la Aplicación Web al realizarse las pruebas manuales en diferentes dispositivos tecnológicos.

Tabla 4. Subcriterios de la Adaptabilidad.

\begin{tabular}{lll}
\hline CRITERIO & SUBCRITERIO & PUNTAJE \\
\hline Adaptabilidad & Responsivo & 1 Cumple \\
\cline { 2 - 2 } & Etiqueta ViewPort & 0 No cumple \\
\hline
\end{tabular}

Fuente: Elaboración propia.

\section{Resultados.}

Resultados de la medición de usabilidad y portabilidad en la Aplicación Web tradicional.

- Medición de usabilidad Aplicación Web tradicional

La Tabla. 5 y 6 refleja los resultados obtenidos por usuario en la medición de usabilidad a la versión tradicional de la Aplicación Web de servicios de entrega a domicilio.

Tabla 5. Puntaje SUS por usuario en la versión tradicional de la Aplicación Web.

$\begin{array}{llllllllllllll}\text { Usuario } & \mathbf{1} & \mathbf{2} & \mathbf{3} & \mathbf{4} & \mathbf{5} & \mathbf{6} & \mathbf{7} & \mathbf{8} & \mathbf{9} & \mathbf{1 0} & \mathbf{1 1} & \mathbf{1 2} & \mathbf{1 3} \\ \text { Puntuación } & 60.5 & 72.00 & 60.00 & 70.00 & 80.00 & 90.00 & 72.5 & 82.5 & 82.5 & 80.00 & 80.00 & 90.00 & 85.00\end{array}$

Fuente: Elaboración propia.

Tabla 6. Puntaje SUS por usuario en la versión tradicional de la Aplicación Web.

\begin{tabular}{lllllllllllll}
\hline Usuario & $\mathbf{1 4}$ & $\mathbf{1 5}$ & $\mathbf{1 6}$ & $\mathbf{1 7}$ & $\mathbf{1 8}$ & $\mathbf{1 9}$ & $\mathbf{2 0}$ & $\mathbf{2 1}$ & $\mathbf{2 2}$ & $\mathbf{2 3}$ & $\mathbf{2 4}$ & $\mathbf{2 5}$ \\
\hline Puntuación & 80.00 & 87.5 & 95.00 & 77.5 & 77.5 & 92.5 & 75.00 & 87.5 & 82.5 & 82.5 & 80.00 & 85.00
\end{tabular}

Fuente: Elaboración propia.

Ante las indagaciones se obtuvo las siguientes contribuciones por pregunta conociendo que cada punto oscila entre 0 y 4 :

P1-uso de la aplicación: la mayoría (23) se ubicó entre 3 y 4 lo que arroja una aceptación en cuanto a la utilización frecuente de la Aplicación Web tradicional por parte de los 
usuarios; P2.-el sistema es innecesariamente complejo: los usuarios(14) ubican sus contribuciones entre 0 y 1 mostrando una tendencia favorable, en cuanto al resto de las contribuciones se inclinan a una indecisión valorada en 2; P3.- facilidad de uso de la Aplicación: los usuarios (17) favorecen sus contribuciones respecto a la facilidad de la Aplicación Web tradicional; P4.-necesita ayuda de un técnico: la mayoría de los usuarios (20) se inclinan a no necesitar ningún tipo de ayuda técnica, favoreciendo la practicidad de la Aplicación Web; P5.- las funciones del sistema estaban integradas adecuadamente: con mínima diferencia existe una aproximación positiva en cuanto a la cualidad de integración de las funcionalidades (13); P6.- hay mucha inconsistencia en el sistema: las contribuciones de los usuarios (19) manifestaron un desacuerdo con la pregunta, las cuales se ubican entre 0 y 1; P7.- las personas aprenderían a usar este sistema rápidamente: se ubica la mayor parte de los encuestados a la tendencia positiva (16); P8.uso del sistema es muy confuso: al ser una pregunta de carácter negativo las contribuciones de los usuarios (20) se ubican en un rango de 0 y 1 manifestando su desacuerdo con el enunciado; P9.- muy confiado con el uso del sistema: se obtuvo un resultado positivo a pesar de existir solo una mínima diferencia respecto a la mitad de los de más usuarios (14); P10.- aprender un montón de cosas antes de usar apropiadamente el sistema: una de las preguntas mayores puntuadas en donde los usuarios (23) concuerdan en el desacuerdo con el enunciado.

Una vez realizado el cálculo de los resultados generales se obtuvo un promedio de 80.3, valor que interpretado según la Figura 1 ubica a la Aplicación Web tradicional en un rango de usabilidad aceptable, asignándole un adjetivo calificativo Bueno.

\section{- Medición de portabilidad Aplicación web tradicional}

La Tabla. 7 muestra los resultados de las pruebas de adaptabilidad en cinco tipos de dispositivos tecnológicos, los subcriterios de evaluación y el puntaje se obtienen según lo especificado en la Tabla 4.

Tabla 7. Resultado de pruebas manuales de adaptabilidad en diferentes dispositivos tecnológicos.

\begin{tabular}{llll}
\hline & Subcriterios & Dispositivos & Puntaje \\
\cline { 2 - 3 } & Responsivo & Smart TV & 1 \\
\cline { 2 - 3 } & & Ordenadores & 1 \\
\cline { 2 - 3 } & & Tablet & 1 \\
\cline { 2 - 3 } & & Smartphone & 1 \\
\cline { 2 - 3 } & Smartwatch & 0 \\
\hline & & Smart TV & 1 \\
\hline & Ordenadores & 1 \\
\hline & Tablet & 1 \\
\hline & Smartphone & 1 \\
\hline & Smartwatch & 0 \\
\hline
\end{tabular}

Fuente: Elaboración propia. 
En la Tabla. 8 se muestran los resultados conseguidos en cada una las métricas seleccionadas que se relacionan con el parámetro de portabilidad.

Tabla 8. Resultados del cálculo de las métricas de portabilidad.

\begin{tabular}{|c|c|c|c|}
\hline $\begin{array}{l}\text { Factores de la } \\
\text { portabilidad }\end{array}$ & Métricas & Fórmula & Valor de $X$ \\
\hline Adaptabilidad & $\begin{array}{l}\text { Adaptabilidad a } \\
\text { distintos } \\
\text { dispositivos }\end{array}$ & $\mathrm{X}=1-\mathrm{A} / \mathrm{B}$ & $\begin{array}{l}X=1-1 / 5 \\
X=0.8\end{array}$ \\
\hline \multirow[t]{2}{*}{$\begin{array}{l}\text { Capacidad para } \\
\text { ser instalado }\end{array}$} & $\begin{array}{ll}\text { Facilidad } & \text { de } \\
\text { instalación } & \\
\end{array}$ & $\mathrm{X}=\mathrm{A} / \mathrm{B}$ & No Aplica \\
\hline & $\begin{array}{ll}\text { Facilidad } & \text { de } \\
\text { reinstalación } & \end{array}$ & $\mathrm{X}=1-\mathrm{A} / \mathrm{B}$ & No Aplica \\
\hline $\begin{array}{l}\text { Capacidad para } \\
\text { ser reemplazado }\end{array}$ & $\begin{array}{l}\text { Uso continuado de } \\
\text { los datos }\end{array}$ & $\mathrm{X}=\mathrm{A} / \mathrm{B}$ & $X=1$ \\
\hline
\end{tabular}

Fuente: Elaboración propia.

Los valores resultantes en cada una de los subcaracterísticas correspondientes a la portabilidad se reflejan de forma porcentual en la Tabla 9 para una mayor perspectiva del resultado global de la portabilidad de la Aplicación Web Tradicional.

Tabla 9. Factores de portabilidad y su valor porcentual.

\begin{tabular}{l|l} 
Factor de portabilidad & Valor\% \\
\hline Adaptabilidad en dispositivos & $80 \%$ \\
\hline $\begin{array}{l}\text { Adaptabilidad a otros ambientes con } \\
\text { facilidad (amientes similares) }\end{array}$ & $100 \%$ \\
\hline Facilidad de instalación & $0 \%$ \\
\hline Capacidad para ser reemplazado & $100 \%$ \\
\hline Total & $70 \%$ \\
\hline
\end{tabular}

Fuente: Elaboración propia.

Los resultados demuestran que la Aplicación Web tradicional alcanza un valor global del $70 \%$ en portabilidad, dicho porcentaje se obtiene después de calcular las características del parámetro utilizando las métricas y fórmulas definidas por el estándar ISO/IEC.

\section{Resultados de la medición de la Aplicación Web con PWA}

\section{- Medición de usabilidad en la Aplicación Web con PWA}

Los resultados conseguidos mediante el cuestionario SUS aplicado a la medición de usabilidad en la versión de la aplicación web con tecnología PWA, se ve reflejado en la Tabla 10 y 11. 
Tabla 10. Puntuación SUS obtenida por cada usuario en la versión PWA de la Aplicación.

\begin{tabular}{lllllllllllll}
\hline Usuario & 1 & 2 & 3 & 4 & 5 & 6 & 7 & 8 & 9 & 10 & 11 & 12 \\
13
\end{tabular}

\begin{tabular}{llllllllllllll}
\hline Puntuación & 67.5 & 70.00 & 65.00 & 70.00 & 100.00 & 92.5 & 77.5 & 87.5 & 85.00 & 87.5 & 80.00 & 85.00 & 80.00
\end{tabular}

Fuente: Elaboración propia.

Tabla 11. Puntuación SUS obtenida por cada usuario en la versión PWA de la Aplicación

\begin{tabular}{lllllllllllll}
\hline Usuario & 14 & 15 & 16 & 17 & 18 & 19 & 20 & 21 & 22 & 23 & 24 & 25 \\
\hline Puntuación & 92.5 & 92.5 & 90.00 & 92.5 & 80.00 & 100.00 & 77.5 & 92.5 & 92.5 & 75.00 & 85.00 & 80.00
\end{tabular}

Fuente: Elaboración propia.

Ante las indagaciones se obtuvo las siguientes contribuciones por pregunta:

P1-uso de la aplicación: la mayoría (24) se ubicó entre 3 y 4 lo que arroja una aceptación de los usuarios al volver hacer uso de la Aplicación Web de entregas a domicilio; P2.-el sistema es innecesariamente complejo: muestran una tendencia favorable en donde los usuarios (15) resaltan el desacuerdo con la pregunta, en cuanto al resto de las contribuciones se inclinan a una indecisión valorada en 2; P3.- facilidad de uso de la Aplicación: los usuarios (19) favorecen sus contribuciones respecto a la facilidad y practicidad de la Aplicación, los valores oscilan entre 3 y 4; P4.-necesita ayuda de un técnico: la mayoría de los usuarios (22) se inclinan a no ser necesaria; P5.- las funciones del sistema estaban integradas adecuadamente: se valoró positivamente la cualidad de integración de funcionalidad con (13) usuarios a favor; P6.- hay mucha inconsistencia en el sistema: las contribuciones de los usuarios (17) manifestaron un desacuerdo con la pregunta, el resto del puntaje obtuvo un valor contributivo neutro de 2; P7.- las personas aprenderían a usar este sistema rápidamente: se ubica la mayor parte de los encuestados a la tendencia positiva (18); P8.- uso del sistema es muy confuso: al ser una pregunta de carácter negativo las contribuciones de los usuarios (19) se ubican en un rango de 0 y 1 manifestando su desacuerdo con el enunciad; P9.- muy confiado con el uso del sistema: al ubicarse como confianza la posición, resulta positiva la tendencia (22); P10.- aprender un montón de cosas antes de usar apropiadamente el sistema: una de las preguntas mayores puntuadas en donde los usuarios (24) concuerdan en el desacuerdo con el enunciado.

Una vez realizado el respectivo cálculo de los resultados se obtuvo como promedio 84.02, valor que interpretado según Figura 1 ubica a la Aplicación Web con PWA en un rango de usabilidad Aceptable, asignándole un adjetivo calificativo Excelente.

\section{- Medición de portabilidad de la Aplicación Web con PWA}

La Tabla. 12 muestra los resultados de las pruebas de adaptabilidad en cinco tipos de dispositivos tecnológicos, los subcriterios de evaluación y el puntaje obtenido según lo especificado en la Tabla 4. 
Tabla 12. Resultado de pruebas manuales de adaptabilidad en diferentes dispositivos tecnológicos.

\begin{tabular}{|c|c|c|c|}
\hline \multirow{10}{*}{ 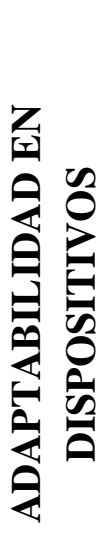 } & Subcriterios & Dispositivos & Puntaje \\
\hline & \multirow[t]{5}{*}{ Responsivo } & Smart TV & 1 \\
\hline & & Ordenadores & 1 \\
\hline & & Tablet & 1 \\
\hline & & Smartphone & 1 \\
\hline & & Smartwatch & 0 \\
\hline & \multirow[t]{5}{*}{ Etiqueta ViewPort } & Smart TV & 1 \\
\hline & & Ordenadores & 1 \\
\hline & & Tablet & 1 \\
\hline & & Smartphone & 1 \\
\hline & & Smartwatch & 0 \\
\hline
\end{tabular}

Fuente: Elaboración propia.

La Tabla. 13 muestra los resultados conseguidos en cada una las métricas seleccionadas que se relacionan con el parámetro de portabilidad.

Tabla 13. Resultados del cálculo de las métricas de portabilidad.

\begin{tabular}{|c|c|c|c|}
\hline $\begin{array}{ll}\text { Factor } & \text { de } \\
\text { portabilidad } & \end{array}$ & Métricas & Fórmula & Valor de $X$ \\
\hline Adaptabilidad & $\begin{array}{l}\text { Adaptabilidad a } \\
\text { distintos } \\
\text { dispositivos }\end{array}$ & $\mathrm{X}=1-\mathrm{A} / \mathrm{B}$ & $\begin{array}{l}X=1-1 / 5 \\
X=0.8\end{array}$ \\
\hline \multirow[t]{2}{*}{$\begin{array}{l}\text { Capacidad para } \\
\text { ser instalado }\end{array}$} & $\begin{array}{ll}\text { Facilidad } \\
\text { instalación }\end{array}$ & $\mathrm{X}=\mathrm{A} / \mathrm{B}$ & $\begin{array}{l}X=1 / 1 \\
X=1\end{array}$ \\
\hline & $\begin{array}{ll}\text { Facilidad } & \text { de } \\
\text { reinstalación } & \end{array}$ & $\mathrm{X}=1-\mathrm{A} / \mathrm{B}$ & $\begin{array}{l}X=1-0 / 1 \\
X=1\end{array}$ \\
\hline $\begin{array}{l}\text { Capacidad para } \\
\text { ser reemplazado }\end{array}$ & $\begin{array}{l}\text { Uso continuado de } \\
\text { los datos }\end{array}$ & $X=A / B$ & $X=1$ \\
\hline
\end{tabular}

Fuente: Elaboración propia.

Los resultados en la Tabla 14 reflejan en valores porcentuales para una mayor perspectiva de la portabilidad para cada una de las métricas seleccionadas. (Ver Tabla 13)

Tabla 14. Factores de portabilidad y su valor porcentual.

\begin{tabular}{l|l}
\hline Factor de portabilidad & Valor\% \\
\hline Adaptabilidad en dispositivos & $80 \%$ \\
\hline $\begin{array}{l}\text { Adaptabilidad a otros ambientes con } \\
\text { facilidad (ambientes similares) }\end{array}$ & $100 \%$ \\
\hline Facilidad de instalación & $100 \%$ \\
\hline Capacidad para ser reemplazado & $100 \%$ \\
\hline Total & $95 \%$ \\
\hline
\end{tabular}

Fuente: Elaboración propia. 
Los resultados demuestran que la Aplicación Web con PWA alcanza un valor del 95\% en portabilidad, dicho porcentaje se obtiene después de calcular las subcaracterísticas del parámetro utilizando las métricas definidas por el estándar ISO/IEC.

\section{Comparación de resultados}

\section{- Medición de usabilidad}

Los valores resultantes del proceso de medición de usabilidad en la Aplicación Web tanto tradicional como PWA se reflejan en la Figura 2.

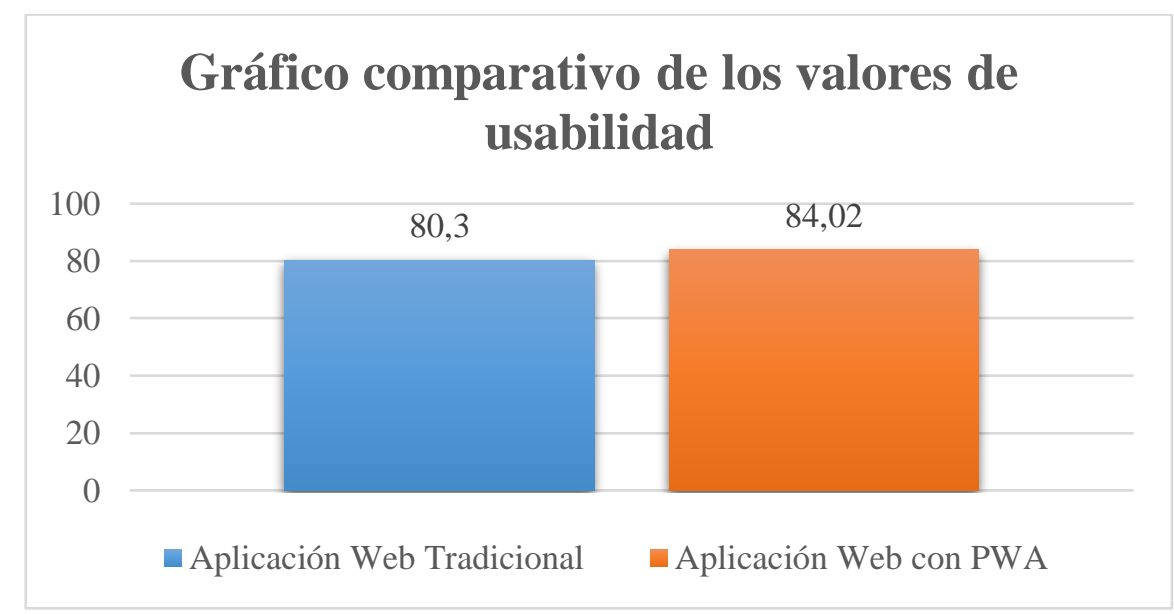

Figura 2 Puntaje SUS final de cada Aplicación Web.

Fuente: Elaboración propia.

De manera general mediante la comparación de los puntajes obtenidos en el test SUS se puede concluir que el parámetro de usabilidad su ubica en un rango aceptable en los dos tipos de aplicaciones web, aunque se presenta una variación mínima que favorece a la Aplicación Web que integra la tecnología PWA.

\section{- Medición de portabilidad}

La Figura 3 muestra los resultados finales conseguidos en la medición de portabilidad tanto en la versión tradicional y progresiva de la Aplicación Web.

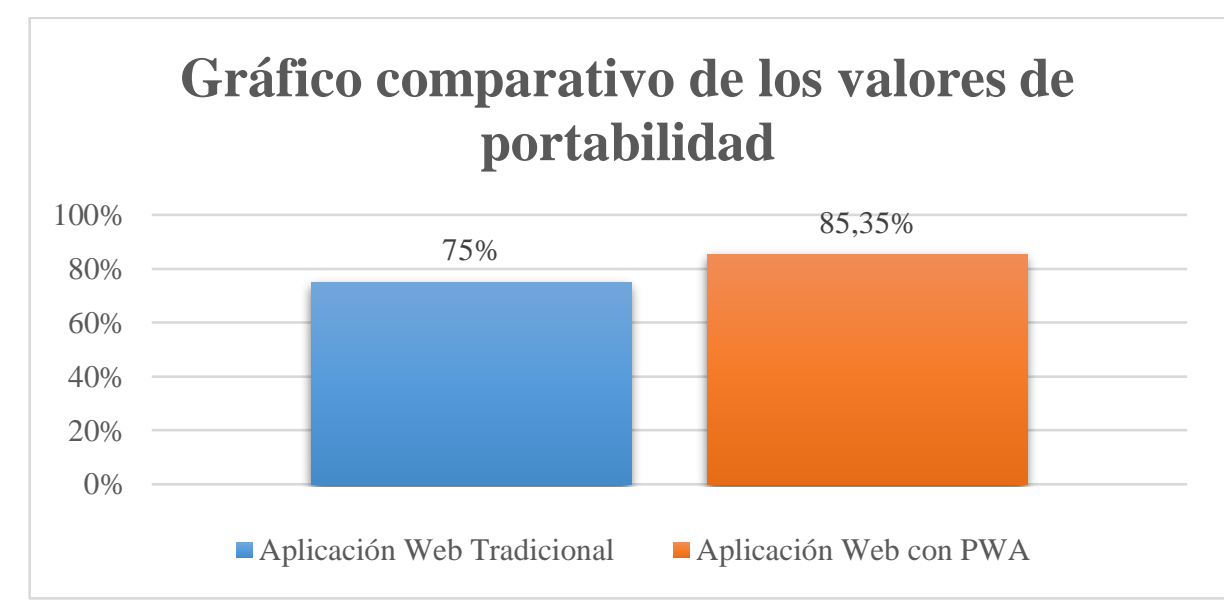

Figura 3 Valor porcentual de portabilidad de cada Aplicación Web.

Fuente: Elaboración propia. 
Como conclusión general se puede observar que la portabilidad en la versión tradicional se aproxima a un $75 \%$ mientras que en la versión con PWA se tiene un valor del $85.25 \%$, esta variación entre las aplicaciones se encuentra principalmente definida por la subcaracterística de instalabilidad relacionada con el parámetro de la portabilidad.

Al realizar un análisis de los resultados del parámetro de Usabilidad se obtuvo que tanto la Aplicación Web tradicional como la Progresiva presentan un puntaje SUS mayor a 80 ubicando a las aplicaciones estudiadas dentro de un rango Aceptable y con adjetivo calificativo Excelente, según la gráfica de clasificación de las puntuaciones del SUS (Ver Figura 1). Los puntajes obtenidos en la Aplicación tradicional y progresiva fueron de 80.3 y 84.02 respectivamente presentado una diferencia mínima equivalente a 3.72 valor que se atribuye a la Aplicación web desarrolla con tecnología PWA, por tanto, favoreciéndose en los resultados. Respecto a las contribuciones recibidas por cada pregunta del cuestionario se presentaron resultados positivos para cada enunciado resaltando para el análisis las preguntas 1, 5 y 10, por su concordancia aproximada en los resultados, P5 hace referencia a la correcta integración de las funciones en la Aplicación Web en donde superándose por mínima diferencia la mitad de los usuarios se inclinaron a una contribución neutra planteando una indecisión, P5 la mayoría de los usuarios concuerdan en no necesitar demasiados conocimientos o habilidades para utilizar las aplicaciones de manera eficiente y alcanzar sus objetivos, y por último P10 considerada clave debido a que la mayoría de los encuestados se inclinan a volver a hacer uso de la Aplicaciones Web analizadas en sus dos versiones.

A pesar que las tecnologías PWA no tienen una gran participación en la elaboración del diseño en las aplicaciones web sus características de rendimiento, reducción de tiempos de carga y notificaciones influyen en la mejora de la experiencia de usuario, cuyo resultado es determinado por el cuestionario SUS denotando una mayor satisfacción, eficiencia y eficacia de las aplicaciones web progresivas referente a las tradicionales.

Respecto al parámetro de portabilidad los resultados obtenidos fueron de $75 \%$ para la aplicación tradicional y $85.35 \%$ para la web progresiva, cabe recalcar que tales porcentajes se basa en la medición de tres subcaracterísticas en que se divide el parámetro (adaptabilidad, capacidad de ser instalado y capacidad de ser remplazado) la diferencia entre los valores se atribuye a la capacidad de instalación que poseen las aplicaciones desarrolladas con PWA, característica que le otorga al usuario la posibilidad de "descargar" la App y añadirla a la pantalla de inicio en su dispositivo tecnológico como si de una aplicación nativa se tratase, determinada característica de instalabilidad brinda una mejor experiencia a usuarios de dispositivos móviles por su capacidad de mantener un diseño adaptable y utilizar funcionalidades propias de una app nativa como las notificaciones push, cualidad que no se atribuye por naturaleza a la aplicación web tradicional. 


\section{Conclusiones.}

- Una vez realizado el proceso de medición del parámetro de usabilidad tanto en la aplicación web tradicional como en la PWA, desarrolladas para el servicio de entrega a domicilio, se obtuvo como resultado puntajes SUS de 80.3 y 84.02 respectivamente, interpretándose con poca diferencia, mejor aceptación de la PWA; el rango de diferencia se atribuye principalmente a las características de las PWA que influyen de forma indirecta en la percepción que el usuario tiene en el uso de aplicación en cuanto a nivel de satisfacción, eficiencia y eficacia.

- Para la característica de portabilidad los resultados fueron de $75 \%$ en la aplicación web tradicional y $85.35 \%$ para la PWA que tiene un mayor porcentaje en la medición. La evaluación de la portabilidad se basó en la medición de sus tres subcaracterísticas (adaptabilidad, capacidad de ser remplazado, capacidad de ser reemplazado) para lo cual se utilizó algunas de las métricas establecidas en la ISO/IEC 9126-3; las pruebas manuales de adaptabilidad se realizaron en cinco tipos de dispositivos tecnológicos (smart TV, ordenadores, tablet, smartphones, smartwatch) obteniendo buenos resultados en las dos versiones de la aplicación web, la capacidad de instalación es el subcriterio que marca la diferencia en los resultados, siendo no aplicable en la aplicación web tradicional.

- La usabilidad de un software es el aspecto que le atribuye universalidad permitiendo que cualquier usuario sin importar su capacidad intelectual utilice el software de manera eficaz y eficiente sin necesitar tantos recursos para llegar a alcanzar su objetivo. La portabilidad por otro lado, brinda esa practicidad al momento de acceder a la aplicación una vez el usuario haya aceptado añadir la App a su dispositivo. La medición de estos parámetros en las dos versiones de la aplicación permitió conocer sus estimaciones y posteriormente realizar el análisis comparativo de los puntajes denotando la percepción de los usuarios ante cada versión, sumándose a esto el resultado positivo alcanzado en la medición de la portabilidad en la Aplicación con PWA.

\section{Referencias bibliográficas.}

Bangor, A., Staff, T., Kortum, P., Miller, J., \& Staff, T. (2009). Determining what individual SUS scores mean: adding an adjective rating scale. Journal of Usability Studies, 4(3), 114-123.

Barbosa, T., Viana, F., Landim, C., Miranda, F., \& Viana, L. (2020). Desarrollo y evaluación de un prototipo de aplicación para cuidadores de ancianos. Revista Electrónica Trimestral de Enfermería, 389-399. https://doi.org/https://doi.org/http://dx.doi.org/10.6018/eglobal.396671.

Bautista Peñaquishpe, A. C. (2019). ESTUDIO DE LAS ARQUITECTURAS TECNOLÓGICAS (APPSHELL, RESPONSIVE DESIGN, SOA) EN LA CONSTRUCCIÓN DE WEB PROGRESIVA PARA FORTALECER LA GESTIÓN DE PEDIDOS DE LOS LOCALES MIPYMES DE TIPO CAFÉ-RESTAURANT EN 
LA CIUDAD DE OTAVALO [Universidad Técnica del Norte]. http://repositorio.utn.edu.ec/handle/123456789/9355

Biørn-hansen, A., Majchrzak, T. A., \& Grønli, T. (2017). Progressive Web Apps : The Possible Web-native Unifier for Mobile Development. Webist, 344-351. https://doi.org/10.5220/0006353703440351

Borsci, S., Federici, S., \& Lauriola, M. (2009). On the dimensionality of the System Usability Scale: A test of alternative measurement models. Cognitive Processing, 10(3), 193-197. https://doi.org/10.1007/s10339-009-0268-9

Geraldes, W., Rodrigues, U., \& Martins, E. (2019). Avaliação da Usabilidade do Scratch utilizando o Método System Usability Scale (SUS) Conference. https://doi.org/10.5753/eri-mt.2019.8589

ISO. (2019). The ISO/IEC 25000 series of standards. The ISO/IEC 25000 Series of Standards, $12, \quad 10 . \quad$ https://iso25000.com/index.php/en/iso-25000standards\%0Ahttps://iso25000.com/index.php/normas-iso-25000/iso-25010/20adecuacion-funcional

Karim, K. (2016). Progressive Web Application Migrating Web Application to a Progressive Web Application. https://play.google.com/store/apps/details?

Khan, A. I., Al-Badi, A., \& Al-Kindi, M. (2019). Progressive web application assessment using AHP. Procedia Computer Science, 155, 289-294. https://doi.org/10.1016/j.procs.2019.08.041

Klug, B. (2017). An Overview of the System Usability Scale in Library Website and System Usability Testing. Weave: Journal of Library User Experience, 1(6), 1-19. https://doi.org/10.3998/weave.12535642.0001.602

Lanza, E. (2019). Aplicación Web Progresiva (PWA) para la gestión de pagos de estacionamiento en superficie [Universidad de Cantabria]. https://repositorio.unican.es/xmlui/bitstream/handle/10902/17089/420138.pdf?sequ ence $=1 \&$ isAllowed $=\mathrm{y}$

Laparra Hernández, J. (2015). Evaluación de la usabilidad web mediante el análisis de la mirada y la respuesta fisiológica. Influencia de las características del usuario [Universidad Tecnológica de Valencia]. https://riunet.upv.es/bitstream/handle/10251/51459/Laparra - Evaluación de la usabilidad web mediante el análisis de la mirada y la respuesta fisiol....pdf?sequence $=1 \&$ isAllowed $=\mathrm{y}$

Lewis Senior HF Engineer, J. R., \& Sauro, J. (2017). Revisiting the Factor Structure of the System Usability Scale. Journal of Usability Studies, 12(4), 183-192.

Mhaske, A., Bhattad, A., Khamkar, P., \& More, R. (2018). Progressive Web App for Educational System. International Research Journal of Engineering and 
Technology, 310-312. https://arc.applause.com/2015/11/30/application-shell-

Mittal, S., \& Kumar Bhatia, P. (2013). Software Component Quality Models from ISO 9126 Perspective: A Review. International Journal of Engineering Sciences, 02(02), 2277-9698.

Mooney, J. (1997). Bringing portability to the software process. Dept. of Statistics and Comp. Sci., West Virginia Univ., Morgantown WV, 559-570. http://ieeexplore.ieee.org/lpdocs/epic03/wrapper.htm?arnumber=5961733

Otzen, T., \& Manterola, C. (2017). Técnicas de Muestreo sobre una Población a Estudio. International Journal of Morphology, 35(1), 227-232. https://doi.org/10.4067/S0717-95022017000100037

Perurena, L., \& Moráguez Bergues, M. (2013). Usabilidad de los sitios Web, los métodos y las técnicas para la evaluación. 24(2), 176-194. https://scielo.isciii.es/pdf/eg/v19n59/1695-6141-eg-19-59-389.pdf

Pressman, R. (2010). Ingeniería del software. Un enfoque práctico (Mc Graw $\mathrm{Hi})$.

Reyes, J. M., Berdugo, M., \& Villegas, L. (2016). Evaluación de usabilidad de un sistema de administración de cursos basado en la plataforma Lingweb. 24, 435-444. https://doi.org/http://dx.doi.org/10.4067/S0718-33052016000300008

Rodríguez, P. (2018). Desarrollo de un cliente web mediante aplicaciones web

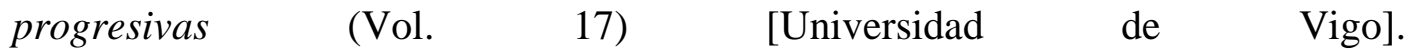
http://castor.det.uvigo.es:8080/xmlui/bitstream/handle/123456789/224/TFG Pablo Rodríguez Pérez.pdf?sequence $=1$

Rodríguez, R. A., Vera, P. M., Martínez, M. R., Alderete, C. G., \& Dogliotti, M. G. (2020). Aplicaciones Web Progresivas Enfocadas en el Uso y Optimización de Cache. 640-644. http://sedici.unlp.edu.ar/bitstream/handle/10915/104215/Documento_completo.pdf ?sequence $=1$

Rodríguez, R. A., Vera, P. M., Martínez, M. R., Parra, F. A., Trigueros, A., \& Dogliotti, M. G. (2019). Aplicaciones Web Progresivas Impulsadas por el Avance de los Estándares Web. http://sedici.unlp.edu.ar/handle/10915/77181

Sensuse, D. I., Satria, D., Pratama, A. A., Wulandari, I. A., Mishbah, M., \& Noprisson, H. (2017). Integrating UCD into Scrumban for better and faster usability design. 2017 International Conference on Information Technology Systems and Innovation, ICITSI 2017 - Proceedings, 2018-Janua(September 2018), 297-302. https://doi.org/10.1109/ICITSI.2017.8267960

Sharma, V., Verma, R., Pathak, V., Paliwal, M., \& Jain, P. (2019). Progressive Web App (PWA) - One Stop Solution for All Application Development Across All Platforms. International Journal of Scientific Research in Computer Science, Engineering and 
Information Technology, 1120-1122. https://doi.org/10.32628/cseit1952290

Steiner, T. (2018). What is in a Web View: An Analysis of Progressive Web App Features When the Means of Web Access is not a Web Browser. The Web Conference 2018 - Companion of the World Wide Web Conference, WWW 2018, 789-796. https://doi.org/10.1145/3184558.3188742

Tandel, S. J. A. (2018). Impact of progressive web apps on web app development. International Journal of Innovative Research in Science, Engineering and Technology, 7(9), 9439-9444. https://doi.org/10.15680/IJIRSET.2018.0709021

Zumba, J. P., \& León, C. A. (2018). Evolución de las Metodologías y Modelos utilizados en el Desarrollo de Software. INNOVA Research Journal, 3(10), 20-33. https://doi.org/10.33890/innova.v3.n10.2018.651

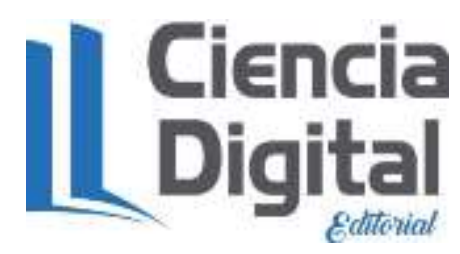




\section{PARA CITAR EL ARTÍCULO INDEXADO.}

Macías Guamangate, Y. K., Miranda Caisaluisa, J. L., \& Tapia Cerda, V. del C. (2021). Medición de usabilidad y portabilidad de una Aplicación Web desarrollada con $\begin{array}{llll}\text { tecnología PWA } \quad \text { ConcienciaDigital, 6-27. } & \text { 4(4), }\end{array}$ https://doi.org/10.33262/concienciadigital.v4i4.1882

\section{LCiencia}

El artículo que se publica es de exclusiva responsabilidad de los autores y no necesariamente reflejan el pensamiento de la Revista Conciencia Digital.

El artículo queda en propiedad de la revista y, por tanto, su publicación parcial y/o total en otro medio tiene que ser autorizado por el director de la Revista Conciencia Digital.

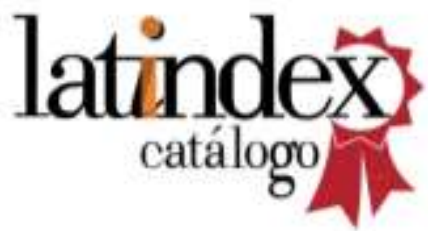

